Achim Geisenhanslüke, Georg Mein (Hg.)

Grenzräume der Schrift

Literalität und Liminalität

hrsg. v. Achim Geisenhanslüke und Georg Mein | Band 2 


\title{
Die ÜBertragung DeR ReinheIT (MARY DOUglas, Friedrich NIETZSCHE)
}

\author{
OLIVER KOHNS
}

Folgt man der Sozialanthropologin Mary Douglas, dann steht die Vorstellung der >Reinheit` bzw. >Reinigung in engem Zusammenhang zu den »rites de passage«, den Ritualen der Schwelle und des Übergangs, mit denen sich die Ethnologie seit Arnold van Genneps gleichnamiger Untersuchung $^{1}$ aus dem Jahr 1909 beschäftigt. In ihrem Buch Purity and Danger zitiert Douglas die Studie van Genneps als Beitrag zu einer allgemeinen anthropologischen Symbolkunde: »Van Gennep shows how thresholds symbolise beginnings of new statutes. Why does the bridegroom carry the bride over the lintel? Because the step, the beam and the door posts make a frame which is the necessary everyday condition of entering a house. $\ll^{2}$

Schon von Beginn an ist die Schwelle damit verdoppelt in die materielle, reale Schwelle und ihren Zwilling, die symbolische und näherhin metaphorische. Die Einsicht van Genneps, so Douglas, habe darin bestanden, diese Verdopplung erkannt zu haben und Rituale der Schwelle als >Übergangsrituale drucksformen der Transformation von einem sozialen Zustand zu einem anderen. Wenn aber die materielle Schwelle im Ritus immer schon nur symbolische Repräsentation einer im Ritual vorgestellten, letztlich sozialen Schwelle ist, dann können problemlos auch andere Objekte als materielle Schwellen zu Zeichen der Schwelle werden. Insofern die Schwelle in der Analyse der »rites de passage« zentral als Metapher für sozialen Übergang verstanden wird, wird ganz im Sinne der aristotelischen Metapherntheorie das Erkennen von Ähnlichkeiten und Analogien zum wich-

1 Vgl. Arnold van Gennep: Les rites de passage, Paris 1909; Neudruck: New York u.a. 1969.

2 Mary Douglas: Purity and Danger. An Analysis of Concepts of Pollution and Taboo [1966], 2. Aufl., London 1969, S. 114. 
tigen Instrument der ethnologischen Schwellenkunde. »The structure of living organisms is better able to reflect complex social forms than door posts and lintels «, ${ }^{3}$ schreibt Mary Douglas. Damit ist sie bei dem zentralen Thema ihrer Untersuchung angekommen: Der menschliche Körper als Medium gesellschaftlicher Symbolik. »The body«, schreibt Douglas, »is a model which can stand for any bounded system. Its boundaries can represent any boundaries which are threatened or precarious. « ${ }^{4}$ Die Grenzen und Öffnungen des Körpers symbolisieren in diesem Modell ebenso wie Grenzen und Schwellen von Häusern, wenn nicht besser als diese - die Übergänge und Schwellen der Gesellschaft. Die kulturellen Vorstellungen von Einheit und Reinheit des menschlichen Körpers interpretiert Douglas sodann als Ausdruck einer kollektiven Imagination: der Angst vor der Bedrohung der Einheit und Reinheit des politischen Körpers. Die Metaphorik der körperlichen und sozialen Reinheit erscheint dann, ebenso wie diejenige der Schwelle, als eine symbolische Ordnung, in der die Gefahr gesellschaftlicher Unordnung »zum Ausdruck gebracht « werden kann. ${ }^{5}$

Die Gemeinsamkeit von >Schwelle und >Reinheit liegt demzufolge in ihrer Funktion, metaphorisch gesellschaftliche Randzonen beschreibbar und diskursiv verfügbar zu machen. Die kulturwissenschaftliche Analyse von Liminalität betreibt in diesem Paradigma eine gewissermaßen angewandte Metaphorologie: Sie erforscht die Konzepte des sozialen Randes, des Übergangs aus einer gesellschaftlichen Ordnung heraus oder in sie hinein. Van Gennep und Mary Douglas bieten mit anderen Worten an, die Konzepte der >Schwelle $<$ und der >Reinheit als »Hintergrundmetaphern ${ }^{6}{ }^{6}$ (Blumenberg) zu interpretieren, mit denen

3 Ebd.

4 Ebd., S. 115.

5 Vgl. Mary Douglas: »Die zwei Körper«, in: dies.: Ritual, Tabu und Körpersymbolik. Sozialanthropologische Studien in Industriegesellschaft und Stammeskultur, übers. v. Eberhard Bubser, Frankfurt a.M. 1981, S. 99-123, hier S. 121: »Wie van Gennep gesehen hat, wird der Übergang von einem sozialen Status in den nächsten immer durch bestimmte materielle Symbole dieses Schritts zum Ausdruck gebracht. Diese Form wird offensichtlich nicht von Kulturschranken beeinflußt und kann deshalb als ein natürliches symbolisches Ausdrucksverhalten verstanden werden. In einer wesentlich tieferliegenden Schicht wird die soziale Erfahrung des saus den Fugen Geratens $₫$, der tiefgreifenden Unordnung in der Gesellschaft, durch extrem wirkungskräftige Symbole für >Unreinheit` und >Gefahr`zum Ausdruck gebracht.«

6 Vgl. Hans Blumenberg: Paradigmen zu einer Metaphorologie [1960], Frankfurt a.M. 1998, S. 91f. 
jeweils das Verlassen (und der Wiedereintritt) bzw. die Auflösung (und die Erneuerung) der politischen und sozialen Ordnung sagbar werden kann. Sowohl >Schwelle< als auch >Reinheit Metaphern für Inklusion und Exklusion, für Ein- und Austritte aus der gesellschaftlichen Ordnung insgesamt.

Körperöffnungen beispielsweise können sowohl als Schwellen wie auch als Orte der Reinheit oder Unreinheit thematisiert werden. Entsprechend wendet Douglas sich ihnen ausführlich zu und interpretiert die rituelle und diskursive Rolle von Körperöffnungen als Ausdruck der Angst vor der Öffnung und Verletzung gesellschaftlicher Ordnung. Die Unreinheit des individuellen Körpers spiegelt in diesem Modell die Unordnung des sozialen Körpers. ${ }^{7}$ So schreibt Douglas:

Any structure of ideas is vulnerable at its margins. We should expect the orifices of the body to symbolise its specially vulnerable points. Matter issuing from them is marginal stuff of the most obvious kind. Spittle, blood, milk, urine, faeces or tears by simply issuing forth have traversed the boundary of the body. [...] To understand body pollution we should try to argue back from the known dangers of society to the known selection of body themes and try to recognise what appositeness is there. ${ }^{8}$

Obwohl Douglas ankündigt, die Körpermetaphorik sei der Vorstellung der Schwelle überlegen, hört sie nicht auf, von Schwellen zu sprechen. Die Schwelle zwischen Körper und Nicht-Körper - die Körperöffnung erscheint hier als hochsensibler Raum des Übergangs, in den soziale Ängste und Gefahren projiziert werden können. Die Verunreinigung, die Verschmutzung durch den Austritt diverser Materien aus dem Körper, kann so als Metapher für das unkontrollierte Überschreiten sozialer Schwellen und den damit verbundenen Verlust gesellschaftlicher Ordnung begriffen werden. Douglas' Interpretation der Körpersymbolik besticht durch ihre Einfachheit und Reichweite: Die Analogie zwischen körperlicher Unreinheit und der Angst vor gesellschaftlicher Unordnung beansprucht universelle Gültigkeit. Winfried Menninghaus kommentiert Douglas' »Modell der Attribution von Unreinheit als Problemlösungsstrategie ${ }^{9}$ in seiner Studie über Ekel, indem er die Möglichkeit erwägt, die »Ästhetik des idealschönen Körpers« auf die Krise einer Ordnung zurückzuführen, »die mittels der Unterscheidung von rein und unrein Am-

7 Vgl. M. Douglas: Purity and Danger, S. 3: »I believe that some pollutions are used as analogies for expressing a general view of the social order.«

8 Ebd., S. 121.

9 Winfried Menninghaus: Ekel. Theorie und Geschichte einer starken Empfindung, Frankfurt a.M. 1999, S. 157. 
bivalenzen und Gefährdungen ihrer Ränder artikuliert und regelt. « ${ }^{10} \mathrm{Die}$ Ästhetik des 18. Jahrhunderts mit ihrem an der antiken Statue orientierten Ideal des makellosen Körpers erscheint dann als Ausdruck einer »instabilen Ordnung voller Ambivalenzen und ungeklärter Hierarchien «. ${ }^{11}$

So "verlockend ${ }^{12}$ diese Applikationen sein mögen: ${ }^{13}$ Sie dürfen nicht vergessen, dass ihre gesamte Autorität auf der Kraft der Ähnlichkeit und Analogie beruht. Dieser Umstand wird jedoch vergessen, sobald sich das Modell sogar eine prognostische Qualität zuschreibt, wenn Douglas etwa behauptet, in einer strikt >geordneten`Gesellschaft, in welcher $\gg$ the sense of outrage is adequately equipped with practical sanctions in the social order, pollution is not likely to arise«. ${ }^{14}$ Spätestens hier zeigt sich, dass die scheinbar nüchterne anthropologische Analyse von kollektiven Ritualen und Vorstellungen von einem durchaus restriktiven Ordnungsmodell angetrieben wird.

Wie gelangen diese Ordnungsvorstellungen in die anthropologische Analyse? Die sozialanthropologische Interpretation der Unterscheidung zwischen rein und unrein als gesellschaftliche Schwellen- und Grenzkategorie führt theologische und rituelle Vorstellungen der Reinheit auf ihre politischen Implikationen zurück. Der politische Subtext, den Douglas >unter ihrem theologischen und ethnologischen Material zu entdecken meint, ist immer derselbe: Eine sich autoritär stabilisierende Ordnung wird einer als destabilisierend beschriebenen Unordnung vorgezogen. Insofern sie »Kultur« als »a positive pattern« definiert, »in which ideas and values are tidily ordered $«,{ }^{15}$ ist Ordnung und sogar Sauberkeit für Douglas letztlich das Prinzip der Kultur überhaupt. Das Prinzip der Ordnung konzipiert Douglas jedoch nach Richtlinien einer autoritären Durchsetzung von Macht, wie sich etwa ausgerechnet in ihren Anmerkungen zum Verhältnis der Geschlechter in der Gesellschaft zeigt. So beschreibt Douglas eine männlich dominierte, nicht gewaltfreie Machtausübung kurzerhand als ideale politische Organisation, in der Vorstellungen von Unreinheit (als rituelle Kompensation politischer Unruhen) gar nicht erst aufkämen. »When male dominance is accepted as a central principle of social organisation and applied without inhibition and with

10 Ebd., S. 152.

11 Ebd.

12 Ebd.

13 Vgl. als weitere Applikation Christian Moser: "»Throw me away<: Prolegomena zu einer literarischen Anthropologie des Abfalls«, in: Archiv für das Studium der neueren Sprachen und Literaturen 242 (2005), S. 318-337.

14 M. Douglas: Purity and Danger, S. 132.

15 Ebd., S. 38f. 
full rights of physical coercion, beliefs in sex pollution are not likely to be highly developed. $«^{16}$

Douglas' Modell partizipiert demnach ungebrochen an einer Ethik und Politik der Reinheit, die autoritäre und puritanische Vorstellungen von Reinheit und Ordnung als soziale Grundwerte festschreibt. Ausdrücklich lobt Douglas den »intelligent, Calvinist approach $«,{ }^{17}$ den Robertson Smith's The Religion of the Semites (1889) nicht nur analysiert, sondern auch selbst vertritt: »For he could show with unrivalled erudition that all primitive religions express social forms and values. $\ll^{18}$ In diesem Sinne versucht auch Douglas mit gleichfalls unbestreitbarer Gelehrsamkeit nachzuweisen, dass das Thema der >Reinheit in allen Religionen und Kulturen der Welt die universelle Wertschätzung sozialer Hierarchie und puritanischer Ordnung zum Ausdruck bringt. Insofern Douglas diese Kategorien und Wertungen durchgehend als überzeitliche und interkulturelle anthropologische Konstanten beschreibt, werden sie letztlich naturalisiert und jeder kritischen Betrachtung entzogen.

Aber nicht nur der politische Inhalt, sondern selbst noch die methodischen Grundlagen von Douglas' Studie gehorchen dem Prinzip der Reinheit. Die Basis für Douglas' Ansatz ist der unverrückbare Glaube an die Analogie zwischen sinnlichen Signifikanten (z.B. Ritualen) und der intelligiblen Sphäre des Signifikats (z.B. einer politischen Idee) - und also letztlich der platonisch-augustinische Dualismus zwischen materieller sinnlicher Welt und reiner intelligibler Idealität.19 Die methodische Grundlage dieser phantasievollen Studie über die Metaphorik der Reinheit ist mit anderen Worten der Glaube an eine reine Metaphorik: an die kontrollierte und ungestörte Übersetzbarkeit von Ritualen und Vorstellungen in die metaphorisch induzierte Gegensätzlichkeit von politischer Ordnung und Unordnung. Die Kategorie der Reinheit greift demnach in Douglas' Studie auf die methodische Vorgehensweise und auf die politischen Inhalte aus. In diesem unkontrollierten Übergreifen zwischen verschiedenen diskursiven Ebenen erweist sie sich als ihrerseits genuin unrein.

16 Ebd., S. 142.

17 Ebd., S. 18.

18 Ebd.

19 Vgl. Jacques Derrida: Grammatologie [1967], übers. v. Hans-Jörg Rheinberger u. Hanns Zischler, 5. Aufl., Frankfurt a.M. 1994, S. 27f.; Kurt Flasch: Augustin. Einführung in sein Denken, 3., erg. Aufl., Stuttgart 2003, S. 320f. Insofern der Dualismus zwischen sinnlichem Signifikanten und idealem Signifikat auch die >abendländische` Philosophie der Schrift (bzw. der Stimme als Phantasma der Identität beider) geprägt hat, liegt in dieser Verbindung von Literalität und Metaphorik zugleich ein systematischer Konnex der Begriffe Literalität und Liminalität. 
Restriktive politische Implikationen können mutatis mutandis auch in diversen Konzeptionen der >Schwelle< beschrieben werden. Van Genneps Modell der Liminalität mit seinen drei Phasen - die rites préliminaires (rites de séparation), liminaires (rites de marge) und postliminaires (rites d'agrégation ${ }^{20}$ - zielt geradezu teleologisch auf eine nur befristete Aussetzung der gesellschaftlichen Ordnung, deren postliminale Wiedererrichtung sich notwendig anschließt. Man kann jedoch auch mit David Wellbery (der sich eher an Victor Turner als an van Gennep orientiert) hervorheben, Liminalität sei »nicht als geregelte Ordnung von Elementen und Relationen, mithin nicht als Struktur zu erfassen, sondern [...] als Anti-Struktur «. ${ }^{21}$ Die »Theorie der Liminalität «, schreibt Wellbery, »behandelt die kulturellen Schwellenphänomene als Überschreitungen, die Struktur selber suspendieren $\aleph^{22}$ - und rückt in diesem Sinn nicht die Ordnung, sondern geradezu im Gegenteil die Aussetzung der Ordnung, die nicht mehr nach dem Modell der Struktur gedacht werden kann, in den Mittelpunkt. Es bleibt jedoch fraglich, ob eine Theorie der Liminalität jede Verbindung zu Struktur und Ordnung von sich weisen kann, solange sie auf das Konzept und die Ordnung der Metapher angewiesen bleibt.

Die Konzepte der >Schwelle〈 und der >Reinheit « versprechen auf je verschiedene Art und Weise den Ausdruck eines Jenseits der sozialen Ordnung, aber ihr tropologischer (bzw. diskursiver) Charakter gehört zum Zentrum dieser Ordnung. Dieser kategoriale Widerspruch zeigt, dass es auch der Sozialanthropologie und Kulturwissenschaft des 21. Jahrhunderts nicht erspart bleiben kann, sich weiterhin mit den aus der sprachkritischen, literatur- und zeichentheoretischen (und also nahezu der gesamten philosophischen) Tradition ererbten Problemen zu beschäftigen. Die Frage, ob und wie es möglich ist, innerhalb sprachlicher Strukturen die Grenzen der sprachlichen Struktur zu überschreiten oder wenigstens aufzuzeigen, ist bereits eines der zentralen Themen der Texte Friedrich Nietzsches. Nicht ohne Grund gilt Nietzsches Philosophie für einige Interpreten als eine zentrale Anregung für das Projekt der >Dekonstruktion $<{ }^{23}$ Im Folgenden soll anhand des Konzepts der >Reinheit< eine doppelte Bewegung in den Texten Nietzsches nachvollzogen werden:

20 Vgl. A. v. Gennep: Les rites de passage, S. 14.

21 Vgl. David E. Wellbery: »Rites de passage. Zur Struktur des Erzählprozesses in E.T.A. Hoffmanns Prinzessin Brambilla«, in: ders.: Seiltänzer des Paradoxalen. Aufsätze zur ästhetischen Wissenschaft, München, Wien 2006, S. 118-145, hier S. 122.

22 Ebd.

23 Vgl. etwa Ernst Behler: Derrida - Nietzsche, Nietzsche - Derrida, München u.a. 1988. 
Die Kategorie wird in mehreren Kontexten scharf kritisiert und gleichzeitig paradoxerweise in ihrer Relevanz uneingeschränkt bestätigt.

Es soll versucht werden, zu zeigen, welche philosophische und rhetorische Dynamik die Texte Nietzsches in der Arbeit an und mit der Kategorie der >Reinheit` entwickeln. Zu diesem Zweck soll zunächst Nietzsches fundamentale Kritik der Kategorie Reinheit in drei verschiedenen Kontexten beschrieben werden (II.). Anschließend soll versucht werden, die >Gegenbewegung zu dieser Kritik in Nietzsches Diskurs nachzuvollziehen und zu erklären, warum Nietzsches eigene Texte nicht weniger als eine gesamte Mythologie der Reinheit entwickeln (III). Zuletzt soll skizzenhaft das Verhältnis dieser paradoxen Bewegung der >Reinheit ' bei Nietzsche im Verhältnis zur >Dekonstruktion` diskutiert werden (IV.).

Die Texte Nietzsches entwickeln die bis dahin wohl eindeutigste und nicht zuletzt systematischste Kritik der Kategorie der Reinheit. Diese Kritik entwickelt Nietzsche in verschiedenen Kontexten: erstens im $\mathrm{Zu}-$ sammenhang mit der genealogischen Kritik der Moral, zweitens im Kontext seiner frühen Rhetorikanalysen und drittens im Zusammenhang mit der epistemologischen Kritik an der Kant'schen Transzendentalphilosophie. Bei näherer Betrachtung wird jedoch zu zeigen sein, dass die Kritik der Reinheit jeweils aus sprach- und rhetoriktheoretischen Überlegungen heraus motiviert wird.

Der erste Kontext, innerhalb dessen Nietzsche eine grundsätzliche Kritik der Kategorie der Reinheit formuliert, ist seine >genealogische Untersuchung moralischer Grundbegriffe. Eine der zentralen Thesen Nietzsches in der Genealogie der Moral (1887), die er in verschiedenen Varianten durchbuchstabiert, ist die grundsätzliche Möglichkeit der Ableitung eines »seelischen Vorrangs-Begriffs« aus einem ursprünglich "politischen Vorrangs-Begriff «. ${ }^{24}$ Eine politisch herrschende Kraft hat, mit anderen Worten, sich selbst einen moralischen Vorrang zugeschrieben und damit die Semantik eines Begriffs besetzen können. Als Beispiel für eine insbesondere von der Religion zum inneren »Vorrangs-Begriff« umgedeutete Kategorie nennt Nietzsche umgehend das Konzept der >Reinheitく. »Im Übrigen«, schreibt Nietzsche,

24 Friedrich Nietzsche: Sämtliche Werke. Kritische Studienausgabe in 15 Einzelbänden, hg. v. Mazzino Montinari u. Giorgio Colli, Berlin, New York, München 1988, Bd. 5, S. 264. 
sei man davor gewarnt, diese Begriffe >rein` und >unrein` nicht von vornherein zu schwer, zu weit oder gar symbolisch zu nehmen: alle Begriffe der älteren Menschheit sind vielmehr anfänglich in einem uns kaum ausdenkbaren Maasse grob, plump, äusserlich, eng, geradezu und insbesondere unsymbolisch verstanden worden. Der $>$ Reine` ist von Anfang an bloss ein Mensch, der sich wäscht, der sich gewisse Speisen verbietet, die Hautkrankheiten nach sich ziehen, der nicht mit schmutzigen Weibern des niederen Volkes schläft, der einen Abscheu vor Blut hat, - nicht mehr, nicht viel mehr! ${ }^{25}$

Nietzsche vollzieht hier eine für seine >genealogische` Untersuchung charakteristische Geste. Das Konzept der >Reinheit` wird als verblichene Metapher (oder Symbolisierung) für einen ursprünglich konkreten und sinnlichen Vorgang gedeutet. Dann wird die konkrete Bedeutung mit dem Ausdruck politischer Herrschaft verbunden: Reinheit fungiert hier als Ausdruck der sozialen Exklusion, der Erhebung einer Gruppe über andere Gruppen. Die »symbolische« Bedeutung des Begriffs >Reinheit wird als die spätere Ideologie dieser Herrschaft, als Ausdruck eines »seelischen« Vorrangs interpretiert. Nietzsche führt diese »seelische« Bedeutung (etwa als $>$ Reinheit des Herzens $\measuredangle$ ) damit auf eine konkrete politische Exklusion einer sozialen Gruppe durch eine andere (die Abneigung gegen die »schmutzigen Weiber des niederen Volkes«) zurück. Die genealogische Untersuchung zielt auf die Enttarnung von Herrschaftsinteressen, die sich als Religion, Ethik und Psychologie verschleiern.

Nietzsche führt in der Genealogie der Moral eine Analyse - eine Zerlegung, Trennung und insofern: Reinigung - der symbolischen und der unsymbolischen ebenso wie der moralischen und der politischen Ebene des Konzepts der Reinheit vor. Das moralische Konzept der >Reinheit und >Unreinheit $८$ erscheint in dieser Perspektive seinerseits als unreine Vermischung. » Jede Verachtung des geschlechtlichen Lebens, jede Verunreinigung desselben durch den Begriff >unrein«", heißt es in Ecce Ho$m o$, , ist das Verbrechen selbst am Leben, - ist die eigentliche Sünde wider den heiligen Geist des Lebens. ${ }^{26}$

Erstaunlicherweise ist die zitierte Passage aus der Genealogie der Moral, wenngleich nicht wörtlich, so doch dem Inhalt nach, bereits in Nietzsches inzwischen berühmt gewordener Basler Rhetorik-Vorlesung (Darstellung der antiken Rhetorik, Sommersemester 1874) angelegt. Nietzsche spricht hier (der zweite Kontext der nietzscheanischen Rede über Reinheit) zwar nicht von moralischer, sondern von sprachlicher Reinheit (puritas) als einem rhetorischen Ideal. Das Schema seines Arguments ist jedoch hier wie dort das gleiche: Es handelt sich jeweils um

25 Ebd., Bd. 5, S. $264 f$.

26 Ebd., Bd. 6, S. 307. 
eine grundsätzlich tropische (d.h. metaphorische) Selbstzuschreibung einer gesellschaftlichen Gruppe, die sich damit gegenüber einer anderen abgrenzen und auszeichnen will. So heißt es in $\S 4$ der Vorlesung:

Von >Reinheit ist nur die Rede bei einem sehr entwickelten Sprachsinn eines Volkes, der vor allem in einer großen Societät, unter den Vornehmen u. Gebildeten sich festsetzt. Hier entscheidet sich, was als provinziell, als Dialekt u. was als normal gilt d.h. >Reinheit ist dann positiv der durch den usus sanktionirte Gebrauch der Gebildeten u. der Gesellschaft, >Unrein` alles, was sonst in ihr auffällt. Also das Nicht Auffällige ist das Reine. An sich giebt es weder eine reine noch eine unreine Rede. ${ }^{27}$

Nietzsche führt hier den gleichen Gestus fort, mit dem er bereits zuvor in der Vorlesung den Begriff der Rhetorik (als intentional gebrauchtes sprachliches Instrument) zugunsten der Idee einer allgemeinen Rhetorizität (als Eigenschaft von Sprache) verschiebt: ${ }^{28}$ Es gebe, so Nietzsche, »gar keine unrhetorische >Natürlichkeit` der Sprache, an die man appelliren könnte: die Sprache selbst ist das Resultat von lauter rhetorischen Künsten [...]: die Sprache ist Rhetorik «. ${ }^{29}$ Wenn es »keine unrhetorische >Natürlichkeit«« in der Sprache gibt, folgt daraus, dass es in ihr auch keine natürliche >Reinheit` geben kann. Die Idee einer >reinen`, von fremden Einflüssen gesäuberten Sprache setzt eine Natürlichkeit der Sprachentwicklung voraus - eine Abwesenheit von Übertragungen und Übersetzungen in jeder Hinsicht. Nietzsches Vorlesung von 1874 zufolge sind Wörter jedoch »an sich u. von Anfang `an`, in Bezug auf ihre Bedeutung Tropen ${ }^{30}{ }^{30}$ d.h. . $u$ eigentliche Bezeichnungen ${ }^{31}$ und also übertragene Wendungen. Die Konzepte >Natürlichkeit` und >Reinheit` sind damit ebenfalls übertragene Wörter: Metaphern, wenn auch Metaphern für Eigentlichkeit und für das Nicht-Metaphorische. Nietzsche bestimmt das Wesen der Sprache als metaphorisch; das Wesen der Metapher wiederum

27 Friedrich Nietzsche: Darstellung der antiken Rhetorik [SS 1874], in: ders.: Werke. Kritische Gesamtausgabe, begründet v. Giogio Colli u. Mazzino Montinari, weitergeführt v. Wolfgang Müller-Lauter u. Karl Pestalozzi, Berlin, New York 1995, Bd. 2/4, S. 413-502, hier S. 428.

28 Vgl. John Bender/David E. Wellbery: »Die Entschränkung der Rhetorik«, in: Aleida Assmann (Hg.): Texte und Lektüren. Perspektiven in der Literaturwissenschaft, Frankfurt a.M. 1996, S. 79-104, hier S. 88f.

29 F. Nietzsche: Darstellung der antiken Rhetorik, S. 425f.

30 Ebd., S. 426.

31 Ebd. 
definiert er über die Metapher der Übertragung: ${ }^{32}$ einerseits physiologisch als »willkürliche Übertragungen ${ }^{33}$ einer Information aus einer kognitiven Sphäre in die andere und andererseits >diskurshygienisch Übertragung aus einer sozialen Situation in eine andere. ${ }^{34}$

Das »Gefühl für die Reinheit«, fährt Nietzsche fort, entwickelt die Gesellschaft »nach unbewußten Gesetzen u. Analogien: eine Einheit, ein einheitlicher Ausdruck wird erreicht: wie einem Volksstamm ein Dialekt genau entspricht, so einer Societät ein als >rein« sanktionirter Stil $\ll .{ }^{35}$ Die »Einheit« und »Einheitlichkeit« eines »Volks« oder einer »Societät« beruht demnach auf nichts anderem als auf einer »Analogie« zwischen der >Einheit` eines Dialekts und der >Reinheit` einer nationalen Sprache und also wiederum auf dem Prinzip der Metapher. ${ }^{36}$ Dass die derart als >rein beschreibbare Sprache keine andere als die Sprache der »Vornehmen $u$. Gebildeten« ist und folglich die >Reinheit` der Sprache das Ergebnis einer unbegründbaren »positiven« Setzung und Machtausübung dieser Gruppe ist, wird durch die naturalisierende Metapher konsequent verschleiert.

Die Rhetorikvorlesung von 1874 legt damit die technische Grundlage für die späteren »genealogischen« Untersuchungen Nietzsches. Grundsätzlich analysiert Nietzsche in beiden Fällen Formen der Übertragung

32 Vgl. Philippe Lacoue-Labarthe: »Der Umweg« [1971], in: Werner Hamacher (Hg.): Nietzsche aus Frankreich, Berlin, Wien 2003, S. 125-163, hier S. $138 \mathrm{f}$

33 F. Nietzsche: Sämtliche Werke, Bd. 1, S. 878.

34 Aus diesem Grund ist nicht nachvollziehbar, warum Ernst Behler Nietzsches Sprachtheorie von Über Wahrheit und Lüge als eine »nicht-repräsentative, rhetorische Konzeption von Kunst und Sprache« interpretiert (Ernst Behler: »Die Sprachtheorie des frühen Nietzsche«, in: Tilman Borsche/Federico Gerratana/Aldo Venturelli (Hg.): >Centauren-Geburten`. Wissenschaft, Kunst und Philosophie beim jungen Nietzsche, Berlin, New York 1994, S. 99-111, hier S. 106). Weitaus eher könnte man von einer rückhaltlosen Verallgemeinerung des Modells der Übertragung (und damit: der Repräsentation) sprechen; an keiner Stelle beansprucht Nietzsche einen $\mathrm{Zu}$ gang zu einem Jenseits der Repräsentation.

35 F. Nietzsche: Darstellung der antiken Rhetorik, S. 428.

36 Vgl. David Martyn: »Borrowed Fatherland: Nationalism and Language Purism in Fichte's Addresses to the German Nation«, in: The Germanic Review 72 (1997), S. 303-315, hier S. 313: »The very idea of the fatherland depends, in other words, on the notion that its language is something that can be more or less pure.« Zum historischen Zusammenhang diverser Projekte der \Sprachreinigung « mit der Genese des Nationalismus vgl. etwa Peter Burke: Languages and Communities in Early Modern Europe, Cambridge 2004, S. 141-159. 
des Konzepts der Reinheit von einer Sphäre in eine andere. Die beiden zitierten >Genealogien « der Reinheit unterscheiden sich jedoch in der Art und Weise der Übertragung. In der Rhetorikvorlesung erscheint sprachliche Reinheit als Metapher für den »durch den usus sanktionirten Gebrauch«, für sprachliche Norm und Normalität also; in der Genealogie der Moral erscheint die moralische Reinheit ausdrücklich als eine »unsymbolische« Übertragung, d.h. weniger als analogische Metapher, sondern vielmehr als eine metonymische Verschiebung von der Bezeichnung bestimmter >puristischer bzw. >asketischer $<$ Lebensformen auf eine vom »Reinen« beanspruchte innere Überlegenheit (und dann erst wieder die Möglichkeit einer weiteren »symbolischen«, d.h. metaphorischen Übertragung, die den eigentlich »unsymbolischen« Ursprung des Konzepts verschleiert).

Den dritten Kontext der Kritik des Konzepts der Reinheit bildet Nietzsches Auseinandersetzung mit der Kant'schen Transzendentalphilosophie. Die Kant'schen Kategorien der »reinen Vernunft« sowie des »reinen Verstandes« werden, vor allem in Also sprach Zarathustra und in den nachgelassenen Notizen Nietzsches, einer scharfen Kritik unterzogen. Nietzsche interpretiert die Kategorie der >Reinheit` in diesem Kontext als eine Verschleierung der interpretierenden Aktivität des erkennenden Subjekts, als die Errichtung einer (nur) scheinbaren Objektivität. So heißt es in einem nachgelassenen Fragment aus dem Zeitraum zwischen November 1887 und März 1888:

Der >Geist`, etwas, das denkt: womöglich gar >der Geist absolut, rein, pur` diese Conception ist eine abgeleitete zweite Folge der falschen Selbstbeobachtung, welche an >Denken glaubt: hier ist erst ein Akt imaginirt, der gar nicht vorkommt, >das Denken jeder Akt dieses Denkens und sonst nichts Anderes seinen Ursprung hat: d.h. sowohl das Thun, als der Thäter sind fingirt. ${ }^{37}$

Die hier formulierte Kritik des reinen Geistes zielt, wie eine andere Notiz belegt, explizit auf die Kant'sche Erkenntnistheorie. So schreibt Nietzsche: »der Kantsche Kriticismus. Der Intellekt stritt sich selbst das Recht ab sowohl zur Interpretation in jenem Sinne als zur Ablehnung der Interpretation in jenem Sinne. ${ }^{38}$ Das Kant'sche Interesse an apriorischen (und nur insofern >reinen $`$ Formen, ${ }^{39}$ die jeder Erkenntnis vorausgehen

37 F. Nietzsche: Sämtliche Werke, Bd. 13, S. 54.

38 Ebd., Bd. 12, S. 147.

39 So heißt es zu Beginn der ersten Auflage der Kritik der reinen Vernunft: »Aus diesem allem ergibt sich nun die Idee einer besondern Wissenschaft, die zur Kritik der reinen Vernunft dienen könne. Es heißt aber jede Er- 
und die das Subjekt bestimmen und limitieren, erscheint in dieser Perspektive als eine Selbstlimitierung, die sich als eine Limitierung durch $a$ priori vorgegebene Formen missversteht. »Der reine Geist «, heißt es in Der Antichrist, »ist die reine Lüge ... $\ll^{40}$

In diesem Sinne polemisiert Nietzsche im Abschnitt »Von der unbefleckten Erkenntniss« in Also sprach Zarathustra gegen die »ReinErkennenden« und nennt diese »empfindsame Heuchler« und »Lüsterne $:^{41}$ Heuchler, weil sie eine Reinheit apriorischer Formen postulierten, wo tatsächlich ihr eigener, »lüsterner« Wille entscheidet. »Oh, ihr empfindsamen Heuchler, Ihr Lüsternen!«, ruft Nietzsches Zarathustra aus: »Euch fehlt die Unschuld in der Begierde: und nun verleumdet ihr drum das Begehren! « ${ }^{42}$ Nietzsches Polemik gegen die >Reinheit` spielt hier epistemologische und sexuelle Konnotationen des Konzepts gegeneinander aus: Die Vertreter des >reinen Erkennens`, so suggeriert die Passage, vermischen Erkennen und Begehren - ihr Erkennen ist tatsächlich unrein, denn es ist von ihrem Begehren gelenkt und verunreinigt. Die Pointe dieser Polemik zielt damit weniger auf die >platonische ২ Körperfeindlichkeit, wie Wolfram Groddeck in seinem Kommentar zu dieser Passage schreibt, ${ }^{43}$ sondern vielmehr auf eine Kritik des reinen Apriori. Die »Rein-Erkennenden«, die das Erkennen auf die apriorischen Formen des Erkennens beschränken wollen, leugnen auf unlautere Weise den Einfluss ihres Willens auf ihr Erkennen.

Die Parallele dieser Argumentation zur genealogischen Kritik des Konzepts der moralischen Reinheit ist unübersehbar. Auch im Feld des epistemologischen Begriffs der Reinheit versucht Nietzsche, das Konzept der >Reinheit` als verschleiernde Tarnung für die Ausübung von Herrschaft und Interesse zu entlarven. Abermals geht es um eine analytische

kenntnis rein, die mit nichts Fremdartigen vermischt ist. Besonders aber wird eine Erkenntnis schlechthin rein genannt, in die sich überhaupt keine Erfahrung oder Empfindung einmischt, welche mithin völlig a priori möglich ist « (Immanuel Kant: Werke in sechs Bänden, hg. v. Wilhelm Weischedel, 5. Aufl., Darmstadt 1983, Bd. 2, S. 62 [KrV, A 11f.]). Die Aufgabe der Kritik der reinen Vernunft ist damit, eine reine Anschauung als reines Erkennen durch ein reines Denken zu bestimmen (vgl. Martin Heidegger: Kant und das Problem der Metaphysik [1929], hg. v. FriedrichWilhelm von Hermann, 6. Aufl., Frankfurt a.M. 1998, S. 43).

40 F. Nietzsche: Sämtliche Werke, Bd. 6, S. 175.

41 Ebd., Bd. 4, S. 156.

42 Ebd., S. 157.

43 Vgl. Wolfram Groddeck: »Zarathustras Poetik des Reinen«, in: Norbert Haas/Rainer Nägele/Hans-Jürgen Rheinberger (Hg.): Kontamination, Eggingen 2001, S. 103-117, hier S. 109. 
Reinigung des Begriffs der Reinheit. In Der Antichrist beklagt Nietzsche die »Vergiftung« der »idealistischen« Philosophie mit einem »Theologen-Instinkt «. ${ }^{44}$ In einem nachgelassenen Fragment spricht Nietzsche entsprechend von der verunreinigenden und verschmutzenden Wirkung der Transzendentalphilosophie: "Seit Kant ist alles Reden von Kunst, Schönheit, Erkenntniß, Weisheit vermanscht und beschmutzt durch den Begriff >ohne Interesse $<.{ }^{45}$

Nietzsches eigener philosophischer >Realismus`zielt auf eine Klärung der >theologischen Anteile in der idealistischen Philosophie, d.h. auf die in seiner Perspektive unhinterfragt gebliebenen Anteile traditioneller Metaphysik und Ethik in dieser Kritik bzw. Neubegründung der Metaphysik. Entsprechend kann Nietzsches Polemik gegen die transzendentale Kategorie der >Reinheit $`$ das Vokabular der moralischen Unreinheit aufbieten. In einem weiteren nachgelassenen Fragment beschreibt Nietzsche das >reine Erkennen Selbstbefriedigung des Weisen, wie ihn sich das Volk denkt, das Abseits und Jenseits des >Rein-Erkennenden`, der ganze sublime Onanismus eines Geistes, dem der gute Wille zur That, zur Zeugung, zum Schaffen in jedem Sinne abhanden gekommen ist $«{ }^{46}$ Nietzsches Texte betreiben somit in ihrer Behandlung der Reinheit eine vollständige Umkehrung der transzendentalistischen Bewertung des Konzepts durch Kant. Kants Kritik der reinen Vernunft setzt Nietzsche in diesem Sinn eine Kritik der unreinen Vernunft entgegen. »Auch Kant hat die contradictio in adjecto >reiner Geist» nicht überwunden $«{ }^{47}$ schreibt Nietzsche: Philosophie erscheint in dieser Perspektive nicht als Zurückhaltung des Subjekts zugunsten einer reinen, überindividuellen Erkenntnis reiner Kategorien, sondern notwendig als Äußerung von Machtinteressen. »Was uns ebenso von Kant, wie von Plato und Leibnitz trennt«, schreibt Nietzsche in der gleichen Notiz aus dem Jahr 1885: »»wir glauben an das Werden, allein auch im Geistigen, wir sind historisch durch und durch. ${ }^{48}$ Nietzsche ersetzt den reinen Geist und das reinen Erkennen folglich versuchsweise durch ein unreines Denken, das sich seiner Ausübung von Wertung und Macht stets bewusst bleibt. Unreines Denken ist demnach bewusst interpretierend und interessegeleitet. »Jeder Glaube an Werth und Würdigkeit des Lebens«, heißt es in Menschliches, Allzumenschliches,

44 F. Nietzsche: Sämtliche Werke, Bd. 6, S. 174.

45 Ebd., Bd. 10, S. 243.

46 Ebd., Bd. 12, S. 146.

47 Ebd., Bd. 11, S. 442.

48 Ebd. 
beruht auf unreinem Denken [...]. Versteht man es, sein Augenmerk vornehmlich auf Ausnahmen, ich meine auf die hohen Begabungen und die reinen Seelen $\mathrm{zu}$ richten [...], so mag man an den Werth des Lebens glauben, weil man nämlich die anderen Menschen dabei übersieht: also unrein denkt. ${ }^{49}$

\section{III}

Nietzsche nimmt damit Douglas' anthropologische Interpretation des Konzepts der >Reinheit $\triangleleft$ als Metapher vorweg. Die rhetorische Struktur des Begriffs erscheint bei Nietzsche jedoch ungleich vielschichtiger und komplexer als in der späteren Sozialanthropologie. Während Douglas von einem einfachen Modell der Metapher ausgeht (rituelle oder imaginative Daten verweisen auf ein immer vergleichbares ideelles Signifikat), fördert Nietzsche mit philologischer Präzision eine nicht leicht überschaubare Vielzahl von Bezügen, Verschiebungen und Übertragungen aller Art zutage. Zugleich ändert sich die Bewertung des Phänomens: Während Douglas sich die metaphorische Interpretation unbefragt zu eigen macht, wird sie bei Nietzsche zu einem grundsätzlichen philosophischen Problem. Was bei Douglas als gesellschaftskonstitutive und damit geradezu als anthropologische Konstante erscheint, wird bei Nietzsche als Verschleierung diskursiver Grenzüberschreitungen (z.B. zwischen Theologie und Epistemologie) fragwürdig. Die metaphorische Konstitution des Konzepts, die Douglas problemlos erscheint, wird für Nietzsche zum Ansatz der Frage nach den Interessen, die die rhetorische Übertragung eines Begriffs motivieren und zugleich verschleiern. Nietzsches Kritik der Reinheit verfährt demgemäß notwendigerweise ihrerseits nach dem Paradigma der Reinheit: Es geht ihr darum, unterschiedliche Kontexte zu markieren, Differenzen einzutragen, Übertragungen als solche sichtbar zu machen. Damit ist die Kritik der Übertragung von Reinheit zugleich selbst nichts anderes als eine Anwendung dieser Übertragung. Wenn Nietzsche etwa, wie bereits zitiert, die »Verunreinigung« des geschlechtlichen Lebens »durch den Begriff >unrein « ${ }^{50}$ bemerkt, dann geschieht ein performativer Widerspruch: Eine Kategorie wird $z u$ gleich unterminiert und weiterhin gebraucht.

Innerhalb dieses Paradigmas ist es problemlos möglich, die Wertungen der Opposition umzukehren und ein >unreines« Denken einzufordern. Einerseits erscheint diese Forderung als der Höhepunkt der nietzscheanischen Kritik der Reinheit, andererseits lässt sie als symmetrische Umkehrung einer Hierarchie die Kategorie der Reinheit an sich vollkommen in-

49 Ebd., Bd. 2, S. 52.

50 Ebd., Bd. 6, S. 307. 
takt. Das allein epistemologisch »unreine Denken«, von dem Nietzsche in Menschliches, Allzumenschliches und in anderen Notizen spricht, kann aus der Perspektive des moralischen Urteils auch als absolut »reines" Denken beschrieben werden, ${ }^{51}$ insofern es von der bei Kant und anderen kritisierten illegitimen und täuschenden Vermischung diskursiver Ebenen Abstand zu halten verspricht.

Aus diesem Grund macht man es sich in der Tat zu leicht, wenn man Nietzsches Sprachkritik mit dem Argument kritisiert, dort werde die Metaphysik der Sprache wider Willen durch eine andere Metaphysik der Sprache perpetuiert. ${ }^{52}$ Im Fall der Kategorie der Reinheit jedenfalls handelt es sich nicht um einen Widerspruch, der Nietzsche versehentlich >unterläuft $\iota$ : Die Kategorie der Reinheit wird emphatisch gebraucht und besetzt. »Nur Ein Gebot gilt dir: sei rein! ${ }^{53}$ heißt es in der Fröhlichen Wissenschaft - und in Ecce Homo bemerkt Nietzsche rückblickend: »Mein ganzer Zarathustra ist ein Dithyrambus auf die Einsamkeit, oder, wenn man mich verstanden hat, auf die Reinheit ... « ${ }^{54}$

Tatsächlich ist vor allem Ecce Homo in einigen Passagen eine Abhandlung über Reinheit. In dem Abschnitt »Warum ich so weise bin« bemerkt Nietzsche unvermittelt: »Und hiermit berühre ich die Frage der Rasse. Ich bin ein polnischer Edelmann pur sang, dem auch nicht ein Tropfen schlechtes Blut beigemischt ist, am wenigsten deutsches. $«{ }^{55}$ Hier scheint auf den ersten Blick die biologistische Idee rassischer Reinheit beansprucht $\mathrm{zu}$ werden. Es gibt allerdings mehr als nur eine Invektive Nietzsches gegen die »verlogene Rassen-Selbstbewunderung und Un-

51 Vgl. ebd., Bd. 8, S. 331: »Wer den Trieb zur Reinlichkeit auch im Geistigen hat, wird es nur eine Zeit lang in den Religionen aushalten und sich dann in eine Metaphysik flüchten; später wird er sich von Stufe zu Stufe auch der Metaphysik entschlagen. Es ist wahrscheinlich, dass der Trieb zur Reinlichkeit im Moralischen eher einen entgegengesetzten Weg einschlagen wird; dafür ist dieser Trieb immer mit der Unreinheit des Denkens verbunden und macht dieses vielleicht immer unreinlicher.«

52 Vgl. Jean-Luc Nancy: «)Unsre Redlichkeit!〈 (Über Wahrheit im moralischen Sinn bei Nietzsche)« [1980], in: W. Hamacher: Nietzsche aus Frankreich, S. 225-248, hier S. 229: »Sie wissen recht gut, daß es nun leicht ist, Nietzsche >in die Ecke zu treiben`, da er ja nur im Namen einer weiteren Entsprechungs-Wahrheit oder besser: der immer identischen einen Entsprechungs-Wahrheit sprechen kann. Sie wissen auch, Heidegger hat es gezeigt, daß der, der auf diese Weise versucht, die Wahrheit über Nietzsche zu sagen - um ihn zu denunzieren -, seinerseits selbst Anspruch auf die Wahrheit dessen erhebt, was er über Nietzsche sagt. [...] Lassen wir das also.«

53 F. Nietzsche: Sämtliche Werke, Bd. 3, S. 367.

54 Ebd., Bd. 6, S. 276.

55 Ebd., S. 268. 
zucht $[. .$.$] , welche sich heute in Deutschland als Zeichen deutscher Ge-$ sinnung zur Schau trägt $\ll,{ }^{56}$ und in der Tat geht es hier nicht um eine biologistisch gedachte Reinheit. Indem er sich zu einem »polnischen Edelmann pur sang« erklärt, schreibt Nietzsche seinen eigenen Familienroman, in dem er vor allem mit seiner biologischen Mutter und Schwester nicht verwandt ist: »mit solcher canaille mich verwandt zu glauben wäre eine Lästerung auf meine Göttlichkeit«. ${ }^{57}$ Das pur sang, das Nietzsche sich selbst zuschreibt, ist demnach gerade nicht biologisch zu verstehen. Darauf verweist auch die Fortsetzung der zitierten Passage:

Aber auch als Pole bin ich ein ungeheurer Atavismus. Man würde Jahrhunderte zurückzugehn haben, um diese vornehmste Rasse, die es auf Erden gab, in dem Masse instinktrein zu finden, wie ich sie darstelle. Ich habe gegen Alles, was heute noblesse heisst, ein souveraines Gefühl von Distinktion, - ich würde dem jungen deutschen Kaiser nicht die Ehre zugestehn, mein Kutscher zu sein. ${ }^{58}$

Die Selbstzuschreibung der Reinheit wird hier offen als eine »Distinktion« markiert: als Auszeichnung, Hervorhebung, Exklusion des eigenen Ich aus der biologischen Familie, der deutschen Nationalität und auch aus der zeitlichen Gegenwart überhaupt (»Man würde Jahrhunderte zurückzugehn haben«). Zugleich wird hier von der Reinheit des Blutes zur Reinheit des Instinktes übergegangen - welcher, wie Nietzsche einige Seiten weiter präzisiert, vor allem ein Instinkt für Reinheit und Reinlichkeit ist:

Mir eignet eine vollkommen unheimliche Reizbarkeit des ReinlichkeitsInstinkts, so dass ich die Nähe oder - was sage ich? - das Innerlichste, die 〉Eingeweide $<$ jeder Seele physiologisch wahrnehme - rieche ... Ich habe an dieser Reizbarkeit psychologische Fühlhörner, mit denen ich jedes Geheimniss betaste und in die Hand bekomme: der viele verborgene Schmutz auf dem Grunde mancher Natur, vielleicht in schlechtem Blut bedingt, aber durch Erziehung übertüncht, wird mir fast bei der ersten Berührung schon bewusst. ${ }^{59}$

Nietzsches »Reinlichkeits-Instinkt« ist demnach ein Instrument der Distinktion in zweierlei Hinsicht: Einerseits führt es zur Zuschreibung von »verborgenem Schmutz« und »schlechtem Blut« bei anderen, andererseits

56 Ebd., Bd. 3, S. 630.

57 Ebd., Bd. 6, S. 268. Vgl. zur Interpretation dieser Passage als »Familienroman« ausführlich: Sarah Kofman: »A Fantastical Genealogy: Nietzsche's Family Romance«, in: Peter J. Burgard (Hg.): Nietzsche and the Feminine, Charlottesville, Va. 1994, S. 35-52.

58 F. Nietzsche: Sämtliche Werke, Bd. 6, S. 268.

59 Ebd., S. 275. 
fungiert er als Zuschreibung eigener Überlegenheit. Dieser reine Instinkt für Reinlichkeit entspricht demnach dem, was Nietzsche in Menschliches, Allzumenschliches als »unreines Denken« beschrieben hat: Sinn für Wertung, für Polemik, Anstreben von Überlegenheit, Exklusion und Distinktion.

Die Suche nach »verborgenem Schmutz« führt in Ecce Homo zielstrebig zu einer erneuten Polemik gegen die Philosophen des >deutschen Idealismus ( oder besser: diejenigen Autoren, die Nietzsche unter diesem Etikett versammelt): »Der >deutsche Geist»«, schreibt Nietzsche, »ist meine schlechte Luft: ich athme schwer in der Nähe dieser Instinkt gewordenen Unsauberkeit in psychologicis, die jedes Wort, jede Miene eines Deutschen verräth. $«{ }^{60}$ In einer nachgelassenen Notiz aus dem Jahr 1885 vermerkt Nietzsche über die »deutschen Idealisten«: »Sie mögen an ihrem Leibe schon die Reinlichkeit lieben: aber ihr Geist ist ungewaschen $\ll .{ }^{61}$ Nietzsches Formel des »Reinlichkeits-Instinkts « ist eine metaphorische bzw. mythologische Inszenierung seiner philosophischen Methodik: der genealogischen Kritik. Diese ist, wie bereits erwähnt, eine Methode der Aufspürung von verschleierten Grenzüberschreitungen philosophischer Begriffe, von philosophischen Unreinheiten: Geschichte erscheint in der Perspektive des genealogischen Verfahrens, wie Friedrich Kittler formuliert, »als Serien [sic!] von Verboten und Übertretungen, Kämpfen und Spannungen ${ }^{62}$

Die Formel des »Reinlichkeits-Instinkts« inszeniert metaphorisch die philosophische Methodik Nietzsches, aber zugleich steht sie im Mittelpunkt einer mythologischen Inszenierung der Autorfigur Nietzsche. In dieser Mythologie ist »Philosophie [...] das freiwillige Leben in Eis und Hochgebirge - das Aufsuchen alles Fremden und Fragwürdigen im Dasein «, ${ }^{63}$ eine »große, immer größere Loslösung, ein willkürliches In-dieFremde-gehen, eine `Entfremdung〈, Erkältung, Ernüchterung $«^{64}-$ »ein

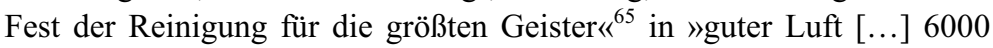
Fuss über Bayreuth «. ${ }^{66}$ In Nietzsches Selbstmythologisierung lebt der Autor in einer Welt, in der die »Luft dünn und rein [ist], die Gefahr nahe - und der Geist voll einer fröhlichen Bosheit: so paßt es gut zu einan-

60 Ebd., S. 361.

61 Ebd., Bd. 11, S. 465.

62 Friedrich A. Kittler: »Wie man abschafft, wovon man spricht: Der Autor von 〉Ecce Homo«« [1980], in: Jacques Derrida/Friedrich Kittler: Nietzsche - Politik des Eigennamens. Wie man abschafft, wovon man spricht, Berlin 2000, S. 65-99, hier S. 197.

63 F. Nietzsche: Sämtliche Werke, Bd. 6, S. 258.

64 Ebd., Bd. 11, S. 664.

65 Ebd., S. 219.

66 Ebd., Bd. 6, S. 270. 
der «. ${ }^{67}$ Das Hochgebirge schließt die zeitgenössische Klimatheorie - Michelet schreibt in »La Montagne« (1868), im Hochgebirge könne man, weit über der niederen Welt, frei atmen ${ }^{68}-$ mit dem topos >Höhenkammく kurz, der von Petrarca bis in die Gegenwart die Beschäftigung mit >großer< Literatur und >großen macht. ${ }^{69}$ Neben dem Hochgebirge schätzt der Autor Nietzsche nach eigener Auskunft allenfalls noch die Orte, "wo man überall Gelegenheit hat, aus fliessenden Brunnen zu schöpfen (Nizza, Turin, Sils); ein kleines Glas läuft mir nach wie ein Hund «. ${ }^{70}$ Diesen Mythologemen der Reinheit stehen auf der anderen Seite Phantasmen des Schmutzes und der Ansteckung gegenüber. ${ }^{71}$ In Der Antichrist heißt es über die Bibel:

Was folgt darauss? Dass man gut thut, Handschuhe anzuziehn, wenn man das neue Testament liest. Die Nähe von so viel Unreinlichkeit zwingt beinahe dazu. Wir würden uns >erste Christen` so wenig wie polnische Juden zum Umgang wählen: nicht dass man gegen sie auch nur einen Einwand nöthig hätte ... Sie riechen beide nicht gut. ${ }^{72}$

In den späteren Texten und Notizen erscheint insbesondere der Diskurs der Wagnerianer (die »Bayreuther Blätter«) für Nietzsche als »ein

67 Ebd., Bd. 10, S. 73.

$68 »$ La vie nous paraissait légère. Etait-ce l'effet de l'air (à cette hauteur de 2400 pieds)? Etait-ce le dégagement de l'existence inférieure, des pensées d'un monde absent? [...] Je pensai aux amis absents, à la société languissante des grandes villes du bas-pays, de Seine ou du Rhin, de Hollande, aux épais brouillards de Londres. Je me disais, au moment surtout des jolies éclaircies: quel avantage de monter! que le monde n'est-il ici, allégé et affranchi! [...] Lieu de liberté véritable! Plus bas, plus haut, on respire moins « (Jules Michelet: »La Montagne« [1868], in: ders.: Cuvres complètes, 21 Bde., hg. v. Paul Viallaneix, Paris 1971-1987, Bd. 20, S. 89-216, hier S. 97); vgl. Michel Serres: ")Der Antichrist<: Eine Chemie der Begriffe und Empfindungen«, in: ders.: Verteilung. Hermes IV [1977], übers. v. Michael Bischoff, Berlin 1993, S. 182-203, hier S. 188.

69 Vgl. Rembert Hüser: »Frozen Fritz«, in: Michael Jeismann (Hg.): Obsessionen. Beherrschende Gedanken im wissenschaftlichen Zeitalter, Frankfurt a.M. 1995, S. 116-153.

70 F. Nietzsche: Sämtliche Werke, Bd. 6, S. 281.

71 Vgl. M. Serres: Der Antichrist, S. 183: »Es handelt sich um ein Vademekum der Mikrobiologie. [...] Der Text [Nietzsches Der Antichrist, O. K.] gehört dem Pasteurschen Zeitalter an. [...] Es handelt sich also nicht um eine virulente Beschreibung des Christentums, sondern um die phantastische Beschreibung des Verhaltens und der Aktivitäten der virulenten Viren.«

72 F. Nietzsche: Sämtliche Werke, Bd. 6, S. 223. 
Sumpf: Anmaaßung, Unklarheit, Unwissenheit und - Geschmacklosigkeit durcheinander «; ${ }^{73}$ die Gegenwart steht unter der »Herrschaft des Gedankens des demokratischen Zeitgeistes und seiner feuchten Luft «. ${ }^{74}$ Nietzsches Mythologie der Reinheit kreist um die Idee der Unabhängigkeit von der eigenen Zeit: Sie inszeniert eine Selbstexklusion der Autorfigur aus den umgebenden Diskursen. »Nach dem Grade der Unabhängigkeit von Ort und Zeit nimmt die noblesse zu«, erklärt Nietzsche seine diskursive Strategie in einer nachgelassenen Notiz: »Menschen der höchsten Cultur, aus starken Leibern, stehen über allen Souveränen. ${ }^{75}$ In einer anderen Notiz heißt es bündig: »Ich will nicht vermischt und verwechselt sein. ${ }^{76}$

Um jede Vermischung und Verwechslung zu vermeiden, betreibt Nietzsches diskursive Unabhängigkeitserklärung wesentlich die Exklusion anderer diskursiver Autorinstanzen - vor allem der Namen Richard Wagners und Schopenhauers. Beiden hatte Nietzsche jeweils ein ganzes Buch gewidmet, welche, wie er nun in Ecce Homo verkündet, eigentlich Bücher über ihn selbst gewesen seien: Man dürfe, schreibt Nietzsche in seiner Schrift ») Wagner in Bayreuth ‘[...] rücksichtslos meinen Namen oder das Wort >Zarathustra< hinstellen, wo der Text das Wort Wagner giebt «, ${ }^{77}$ und die Schrift Schopenhauer als Erzieher tauft Nietzsche hier nachträglich in Nietzsche als Erzieher ${ }^{78}$ um. Noch weiter treibt Nietzsche seine diskursive Unabhängigkeit durch die Behauptung radikaler Askese in der Aufnahme fremder Gedanken überhaupt. So heißt es in Ecce Ho$m o$ : »Werde ich es erlauben, dass ein fremder Gedanke heimlich über die Mauer steigt? - Und das hiesse ja lesen ... [...] Ich muss ein Halbjahr zurückrechnen, dass ich mich mit einem Buch in der Hand ertappe. ${ }^{79}$

Wie der gesamte Text von Ecce Homo (und wie viele andere Texte Nietzsches) bewegen sich diese Formulierungen stets hart an der Grenze zum Übertriebenen und Überzogenen. ${ }^{80}$ Offenkundig muss jedes Mittel

73 Ebd., Bd. 11, S. 254.

74 Ebd., S. 439.

75 Ebd., S. 82.

76 Ebd., Bd. 10, S. 410.

77 Ebd., Bd. 6, S. 314.

78 Ebd., S. 320.

79 Ebd., S. 284. „So ist Nietzsche unter gebildeten, und das heißt abwechselnd lesenden und schreibenden Individuen einzig und individuell darin, daß er taub und blind ist. Er liest nicht mehr, er schreibt nur.« (F. Kittler: Wie man abschafft, wovon man spricht, S. 74).

80 »Nietzsches Schreiben ist irreduzibel hyperbolisch«, schreibt Alexander Nehamas nicht zu Unrecht (Alexander Nehamas: Nietzsche. Leben als Literatur, übers. v. Brigitte Flickinger, Göttingen 1996, S. 43). 
genutzt werden, um die Distinktion des eigenen Diskurses zu suggerieren, die Gefahr der Verunreinigung desselben (durch die Anerkennung anderer diskursiver Instanzen, durch die Aufnahme »fremder Gedanken«) erscheint hoch. »Im reinsten Quell ist ein Tropfen Schmutzes genug $-\ll,{ }^{81}$ schreibt Nietzsche beunruhigt in sein Notizbuch.

Deutlich erkennbar ist zudem, dass Nietzsche seine moralische »noblesse « und seine diskursive Autorität nicht anders vor Verunreinigungen zu schützen vermag als durch eine aufwendige Mythologisierung und Fiktionalisierung seines eigenen Diskurses. Der »Trieb zur Reinlichkeit im Moralischen«, notiert Nietzsche bereits 1876, sei »immer mit der Unreinheit des Denkens verbunden $« .{ }^{82}$ Der »Reinlichkeits-Instinkt « und die durch diesen inszenierte philosophische Souveränität fällt in den Bereich des »unreinen Denkens« und kann jederzeit von Nietzsches eigener genealogischer Methode als >unlautere Fiktion, als Vermischung diskursiver Ebenen (etwa: von Psychologie und Selbstmythologisierung) kritisiert werden.

Nietzsches Kritik und sein affirmativer Gebrauch der Kategorie >Reinheit` verschränken sich hier bis zur Ununterscheidbarkeit. Der »Reinlichkeits-Instinkt«, von dem Nietzsche in Ecce Homo spricht, ist eine Beschreibung seiner philosophischen Methodik, mit der er nicht zuletzt verschiedene Diskurse der Reinheit kritisiert und entmystifiziert hat, - und er ist zugleich selbst ein Element einer solchen Mystifizierung, die von der Kategorie >Reinheitく scheinbar unwiderstehlich ausgeht. »Die Falschheit eines Begriffs «, bemerkt Nietzsche in einer nachgelassenen Notiz, »ist mir noch kein Einwand gegen ihn. Darin klingt unsere neue Sprache vielleicht am fremdesten: die Frage ist, wie weit er lebenfördernd, lebenerhaltend, arterhaltend ist. ${ }^{83}$ In diesem Sinn ist auch das Konzept der >Reinheit` für Nietzsche ein Begriff, der trotz seiner Falschheit eine diskursive Funktion übernimmt, weil er »moralische« (und das heißt: politische, nicht zuletzt vor allem diskurspolitische) Intentionen zu erfüllen verspricht. Da jedoch noch die >neutralste` Beschreibung der philosophischen Methode Nietzsches (und vielleicht: der philosophischen Methode überhaupt) nicht ohne die Kategorie >rein kann, ist diese herausgehobene Verwendung nicht allein einer spezifischen Intention Nietzsches zuzuschreiben, sondern auch der Redlichkeit ${ }^{84}$ auf bestimmte »Falschheiten« nicht verzichten zu können.

81 F. Nietzsche: Sämtliche Werke, Bd. 12, S. 35.

82 Ebd., Bd. 8, S. 331.

83 Ebd., S. 526f.

84 Vgl. J.-L. Nancy: Unsre Redlichkeit!, S. 238f.: »Werte schaffen, das heißt am Ende die Notwendigkeit der Welt erschaffen - und neuschaffen -, das heißt sich mit ihrem Gesetz als der Wertung selber, als der wahren Physik 
Der Vergleich zwischen Mary Douglas' und Friedrich Nietzsches Auseinandersetzung mit dem Problem der $>$ Reinheit $`$ zeigt die ungleich höhere Komplexität und Dynamik der Kategorie in den Texten des deutschen Philosophen. Die sozialanthropologische Beschreibung der >Reinheit< versucht, der Kategorie als Metapher eine eindeutige Lesbarkeit zuzuschreiben. Insofern diese Beschreibung jedoch unter der Hand selbst mit einer puritanischen Moral und einer Politik der Reinheit operiert, zeigt sich ein Übersprung des Objekts auf die Ebene des Betrachtenden, der in Douglas' theoretischen Überlegungen nicht vorgesehen und nicht mehr beschreibbar ist. In den Reflexionen Nietzsches zu dieser Thematik erscheint die jederzeitige Möglichkeit der Übertragung der Kategorie $>$ Reinheit in das eigene Sprechen dagegen als eine unausweichliche Folge der rhetorischen Dynamik von Sprache überhaupt. Die Semantik der Reinheit erweist sich hier als ein Phänomen der Übertragung in jeder Hinsicht: Es handelt sich um übertragene Sprache nicht allein im Sinn des Metaphorischen, sondern auch als Übertragung zwischen sprachlichen Bereichen, zwischen diskursiven Ebenen, als Ansteckung der Aussage durch das Ausgesagte. Es handelt sich demnach jederzeit um eine sowohl metaphorische als auch kontagiöse Übertragung: Ein vollkommen >reines` Sprechen über Reinheit erweist sich als unmöglich. Es kann keine »Theorie« der Reinheit geben, die den Gegenstand eindeutig auf objektiver Distanz zu halten vermöchte. ${ }^{85}$

In dieser Anerkennung der Unmöglichkeit einer `Meta-Sprache` und in diesem obsessiven Interesse an den Elementen, die diskursive und logische Grenzen - und noch die Idee der Grenze selbst - überqueren und transformieren, erweisen sich Nietzsches Überlegungen zum Thema >Reinheit als inspirierend für die Philosophie der `Dekonstruktion<. In diesem Sinn schreibt Derrida »Theorie « bewusst in Anführungszeichen: Nicht, um »einen allzu unreinen (impur) Begriff auf Distanz zu halten«, sondern im Gegenteil als »eine Geste des Mißtrauens einem Begriff gegenüber, der frei (pur) von jeder Ansteckung wäre und erfüllt von einer eigentlichen Bedeutung (sens propre), die sich absolut wiederan-

und Physiologie der Wertung identifizieren. Redlichkeit ist Unterwerfung unter die Notwendigkeit dieser wertenden physis; oder genauer: darin liegt die Wirkung der Redlichkeit./Denn Redlichkeit als solche, als Tugend und Wirksamkeit dieser Tugend, ist diejenige Wertung, die die wertende und gesetzgebende physis anerkennt."

85 Dies gilt, mit einigen Variationen und Differenzen, wohl auch für eine jede $»$ Theorie« der 〉Schwelle«. 
eignen ließe «. ${ }^{86}$ Als verunreinigende Kreuzung jeder begrifflichen Differenzierung erscheint in Derridas Texten insbesondere die Figur des Parasiten: >Dekonstruktion〈 sei »als Diskurs stets ein Diskurs über den Parasiten, sie selbst ein parasitäres Dispositiv über den Parasiten «, ${ }^{87}$ bemerkt Derrida in einem Interview. »Parasitär« ist das in einer metaphysischen Hierarchie Herabgestufte und Ausgeschlossene (etwa das >übertrageneく Sprechen in Austins Sprechakttheorie): etwas, was sich am Rande einer Ordnung befindet (und was eine >dekonstruktiveく Lektüre dennoch möglicherweise als eigentliches Zentrum der Struktur erweisen würde). Die Formel des »parasitären Dispositivs über den Parasiten« beschreibt das Eingeständnis, dass das jeweils als parasitär Ausgeschlossene noch im Sprechen über das Parasitäre wirksam sein muss. Das »Parasitäre« erweist sich demzufolge als Exempel eines Begriffs, der seinerseits alles andere als »frei von jeder Ansteckung« ist.

Die aus der Überzeugung des common sense erwachsene Forderung nach >sauberer Definition der Begrifflichkeit - gestärkt durch den Glauben, die Übertragbarkeit eines Konzepts zumindest theoretisch eingrenzen zu können - erscheint in dieser Perspektive als die Verleugnung des unvermeidlichen parasitären Anteils an sprachlichen Funktionen. In der >Anerkennung torischen Hinweis auf die Unmöglichkeit diskursiver >Reinheit` zeigt sich >Dekonstruktion` immer wieder Nietzsches Philosophie der Übertragung und Ansteckung verpflichtet. Wie heißt es in Nietzsches Also sprach Zarathustra? »Und wer unter Menschen nicht verschmachten will, muss lernen, aus allen Gläsern zu trinken; und wer unter Menschen rein bleiben will, muss verstehn, sich auch mit schmutzigem Wasser zu waschen. $\ll^{88}$

86 Jacques Derrida: Einige Statements und Binsenweisheiten über Neologismen, New-Ismen, Post-Ismen, Parasitismen und andere kleine Seismen, übers. v. Susanne Lüdemann, Berlin 1997, S. 30.

87 Jacques Derrida: »Die Rhetorik der Drogen«, in: ders.: Auslassungspunkte. Gespräche, hg. v. Peter Engelmann, übers. v. Karin Schreiner u. Dirk Weissmann, Wien 1998, S. 241-266, hier S. 247. An anderer Stelle notiert Derrida, dass »dieses Wort Parasit mit obsessiver Hartnäckigkeit wiederkehrt, in allem was ich bisher lesen oder schreiben konnte « (Jacques Derrida: »Die Signatur aushöhlen - eine Theorie des Parasiten«, in: Hannelore Pfeil/Hans-Peter Jäck (Hg.): Eingriffe im Zeitalter der Medien, BornheimRoisdorf 1995, S. 29-41, hier S. 31).

88 F. Nietzsche: Sämtliche Werke, Bd. 4, S. 184. 


\section{Literatur}

Behler, Ernst: Derrida - Nietzsche, Nietzsche - Derrida, München u.a. 1988.

Behler, Ernst: »Die Sprachtheorie des frühen Nietzsche«, in: Tilman Borsche/Federico Gerratana/Aldo Venturelli (Hg.): >Centauren-Geburten<. Wissenschaft, Kunst und Philosophie beim jungen Nietzsche, Berlin, New York 1994, S. 99-111.

Bender, John/David E. Wellbery: »Die Entschränkung der Rhetorik«, in: Aleida Assmann (Hg.): Texte und Lektüren. Perspektiven in der Literaturwissenschaft, Frankfurt a.M. 1996, S. 79-104.

Blumenberg, Hans: Paradigmen zu einer Metaphorologie [1960], Frankfurt a.M. 1998.

Burke, Peter: Languages and Communities in Early Modern Europe, Cambridge 2004.

Derrida, Jacques: Grammatologie [1967], übers. v. Hans-Jörg Rheinberger u. Hanns Zischler, 5. Aufl., Frankfurt a.M. 1994.

Derrida, Jacques: »Die Signatur aushöhlen - eine Theorie des Parasiten«, in: Hannelore Pfeil/Hans-Peter Jäck (Hg.): Eingriffe im Zeitalter der Medien, Bornheim-Roisdorf 1995, S. 29-41.

Derrida, Jacques: Einige Statements und Binsenweisheiten über Neologismen, New-Ismen, Post-Ismen, Parasitismen und andere kleine Seismen, übers. v. Susanne Lüdemann, Berlin 1997.

Derrida, Jacques: »Die Rhetorik der Drogen«, in: ders.: Auslassungspunkte. Gespräche, hg. v. Peter Engelmann, übers. v. Karin Schreiner u. Dirk Weissmann, Wien 1998, S. 241-266.

Douglas, Mary: Purity and Danger. An Analysis of Concepts of Pollution and Taboo [1966], 2. Aufl., London 1969.

Douglas, Mary: »Die zwei Körper«, in: dies.: Ritual, Tabu und Körpersymbolik. Sozialanthropologische Studien in Industriegesellschaft und Stammeskultur, übers. v. Eberhard Bubser, Frankfurt a.M. 1981, S. 99-123.

Flasch, Kurt: Augustin. Einführung in sein Denken, 3., erg. Aufl., Stuttgart 2003.

Gennep, Arnold van: Les rites de passage, Paris 1909; Neudruck: New York u.a. 1969.

Groddeck, Wolfram: »Zarathustras Poetik des Reinen«, in: Norbert Haas/Rainer Nägele/Hans-Jürgen Rheinberger (Hg.): Kontamination, Eggingen 2001, S. 103-117.

Heidegger, Martin: Kant und das Problem der Metaphysik [1929], hg. v. Friedrich-Wilhelm von Hermann, 6. Aufl., Frankfurt a.M. 1998. 
Hüser, Rembert: »Frozen Fritz«, in: Michael Jeismann (Hg.): Obsessionen. Beherrschende Gedanken im wissenschaftlichen Zeitalter, Frankfurt a.M. 1995, S. 116-153.

Kant, Immanuel: Werke in sechs Bänden, hg. v. Wilhelm Weischedel, 5. Aufl., Darmstadt 1983.

Kittler, Friedrich A.: »Nietzsche (1844-1900)«, in: Horst Turk (Hg.): Klassiker der Literaturtheorie. Von Boileau bis Barthes, München 1979, S. 191-205.

Kittler, Friedrich: »Wie man abschafft, wovon man spricht: Der Autor von 〉Ecce Homo«« [1980], in: Jacques Derrida/Friedrich Kittler: Nietzsche - Politik des Eigennamens. Wie man abschafft, wovon man spricht, Berlin 2000, S. 65-99.

Kofman, Sarah: »A Fantastical Genealogy: Nietzsche's Family Romance«, in: Peter J. Burgard (Hg.): Nietzsche and the Feminine, Charlottesville, Va. 1994, S. 35-52.

Lacoue-Labarthe, Philippe: »Der Umweg » [1971], in: Werner Hamacher (Hg.): Nietzsche aus Frankreich, Berlin, Wien 2003, S. 125-163.

Martyn, David: »Borrowed Fatherland: Nationalism and Language Purism in Fichte's Addresses to the German Nation«, in: The Germanic Review 72 (1997), S. 303-315.

Menninghaus, Winfried: Ekel. Theorie und Geschichte einer starken Empfindung, Frankfurt a.M. 1999.

Michelet, Jules: »La Montagne« [1868], in: ders.: Euvres complètes, 21 Bde., hg. v. Paul Viallaneix, Paris 1971-1987, Bd. 20, S. 89-216.

Moser, Christian: ॥) Throw me away<: Prolegomena zu einer literarischen Anthropologie des Abfalls«, in: Archiv für das Studium der neueren Sprachen und Literaturen 242 (2005), S. 318-337.

Nancy, Jean-Luc: »Unsre Redlichkeit! (Über Wahrheit im moralischen Sinn bei Nietzsche)« [1980], in: Werner Hamacher (Hg.): Nietzsche aus Frankreich, Berlin, Wien 2003, S. 225-248.

Nehamas, Alexander: Nietzsche. Leben als Literatur, übers. v. Brigitte Flickinger, Göttingen 1996.

Nietzsche, Friedrich: Sämtliche Werke. Kritische Studienausgabe in 15 Einzelbänden, hg. v. Mazzino Montinari u. Giorgio Colli, Berlin, New York, München 1988.

Nietzsche, Friedrich: Darstellung der antiken Rhetorik [SS 1874], in: ders.: Werke. Kritische Gesamtausgabe, begründet v. Giorgio Colli u. Mazzino Montinari, weitergeführt v. Wolfgang Müller-Lauter u. Karl Pestalozzi, Berlin, New York 1995, Bd. 2/4, S. 413-502.

Serres, Michel: »)Der Antichrist $<$ : Eine Chemie der Begriffe und Empfindungen«, in: ders.: Verteilung. Hermes IV [1977], übers. v. Michael Bischoff, Berlin 1993, S. 182-203. 
Wellbery, David E.: »Rites de passage. Zur Struktur des Erzählprozesses in E.T.A. Hoffmanns Prinzessin Brambilla«, in: ders.: Seiltänzer des Paradoxalen. Aufsätze zur ästhetischen Wissenschaft, München, Wien 2006, S. 118-145. 
